\title{
Application of Autonomous Spacecraft Power Control Technology to Terrestrial Microgrids
}

\author{
Timothy P. Dever ${ }^{1}$, Larry M. Trase ${ }^{2}$, and James F. Soeder ${ }^{3}$ \\ NASA Glenn Research Center, Cleveland, OH, 44135, USA
}

\begin{abstract}
This paper describes the potential of the power campus located at the NASA Glenn Research Center (GRC) in Cleveland, Ohio for microgrid development. First, the benefits provided by microgrids to the terrestrial power grid are described, and an overview of Technology Needs for microgrid development is presented. Next, GRC's work on development of autonomous control for manned deep space vehicles, which are essentially islanded microgrids, is covered, and contribution of each of these developments to the microgrid Technology Needs is detailed. Finally, a description is provided of GRC's existing physical assets which can be applied to microgrid technology development, and a phased plan for development of a microgrid test facility is presented.
\end{abstract}

$\begin{array}{ll} & \\ \text { APC } & =\text { Autonomous Power Controller } \\ \text { DER } & =\text { Distributed Energy Resources } \\ \text { ECLSS } & =\text { Environmental Control and Life Support System } \\ \text { EPS } & =\text { Electrical Power System } \\ \text { GRC } & =\text { Glenn Research Center } \\ \text { ISS } & =\text { International Space Station } \\ \text { MM } & =\text { Mission Manager } \\ \text { PSF } & =\text { Power Systems Facility } \\ \text { SNRF } & =\text { Space Network Research Federation }\end{array}$

\section{Introduction}

$\mathrm{M}$ ICROGRIDS, which are smart grids that can be isolated from the main power gird and run autonomously, can provide numerous benefits to the terrestrial power grid. NASA Glenn Research Center (GRC) in Cleveland, Ohio has been working on microgrid development for several years now, with present research efforts directed toward a manned deep space vehicle application. However, the technology developed for space-based microgrids can be applied toward terrestrial microgrid applications, and the physical assets comprising the "power campus" at NASA GRC have the potential to be utilized for development, testing, demonstration, and evaluation of terrestrial microgrid technologies. This Microgrid Test Facility could be used by electrical power utilities and entrepreneurs to test new technologies and equipment in a controlled environment, accelerating the time to market by enabling early demonstration in an operational environment with reduced risk. The Microgrid Test Facility would provide a unique test environment that contains a variety of diverse energy sources, storage elements, and loads that would be present in a robust microgrid of the future. The potential to isolate this entire microgrid from the main power utility will permit edge of the envelope testing and rapid evaluation of innovative control and management strategies. All of these technologies will improve the security of electric power by providing access to local distributed energy resources during grid emergencies, and also enhance economic viability by facilitating the increased availability of inexpensive electric power though the development of distributed microgrid technology on the edge of the present grid.

First, this paper provides a discussion of the benefits of microgrids within the changing power grid. Section III provides an overview of the Technology Needs which must be developed to enable microgrids. Section IV

\footnotetext{
${ }^{1}$ Electrical Engineer, 21000 Brookpark Rd, MS49-8, tdever@nasa.gov.

2 Electrical Engineer, 21000 Brookpark Rd, MS301-5, trase@nasa.gov.

${ }^{3}$ Senior Technologist for Power, 21000 Brookpark Rd, MS301-5, james.f.soeder@nasa.gov.
} 
describes GRC's work in the area of autonomous controller development for microgrids, and details how each of these development efforts impacts the Technology Needs described in Section III. Section V describes GRC's physical assets which could be applied to microgrid development, and Section VI details a plan for phased development of a GRC Microgrid Test Facility.

\section{The Need for Microgrids for $21^{\text {st }}$ Century Power}

The US power grid is facing a number of challenges. Demand is increasing and power flow patterns are evolving, while improved reliability and security remain high priorities. Due to the highly interconnected nature of the grid, changes in one location can impact a large area, and large-scale cascade failures can quickly propagate, with wide-ranging consequences. ${ }^{1} \quad$ The addition of renewable energy sources also poses challenges - regulation requires electricity supply companies to provide a minimum percentage of generation from renewables, which is especially difficult with non-dispatchable sources such as wind and solar. Further complications arise with the addition of increasing numbers of plug-in vehicles, which are becoming a more significant portion of the load. The traditional power grid model, with large remote power stations, long transmission lines, and established load profiles, may be evolving into a new architecture, featuring distributed energy resources (DER, i.e. distributed power generation and energy storage), along with potentially greater flexibility for islanding and microgrids. ${ }^{2}$

This paper focuses on the development of microgrids, which have all of the characteristics of smart grids, and additionally have the ability to be disconnected from the main power grid and run autonomously. Benefits of microgrids include efficiency (lower distribution system loss), reliability (near 100 percent uptime for critical loads), security (enable cyber security and physical security), quality (stable power to meet exacting consumer energy requirements), and sustainability (expand generation to renewables and cleaner fuel sources) ${ }^{3}$.

Microgrids provide a considerable security advantage by allowing islanding, or removing of the microgrid from the main power grid. If power is lost on the main grid for any reason, the microgrid has the assets - distributed energy resources, and an autonomous power control system to detect and manage issues - to seamlessly handle the disconnection from the grid, and provide continued electrical service. Thus essential functions are able to continue uninterrupted, significantly enhancing security for the customer.

Microgrids also provide a solution to the anticipated increases in power demand. With the traditional power grid model, significant increases in power demand require power plant and distribution infrastructure upgrades sufficient to meet peak demand, improvements which would be time consuming and very expensive to implement. Alternately, microgrid technology on the edge of the present grid may be used to reduce congestion on transmission networks at peak times by smart management of DER assets, storing energy and generating power at end of network to meet increasing demand, power that does not have to travel over the long transmission system interconnects that can be crowded during peak use. This will allow the current power grid to handle base load, while microgrids would handle the peak demands, minimizing costly power plant and distribution infrastructure investment.

A notional layout for a microgrid is shown in Figure 1. Note that the typical elements necessary for a smart grid are present: DER, power electronics, a distribution network, and a communication system to enable control of the devices on the grid.

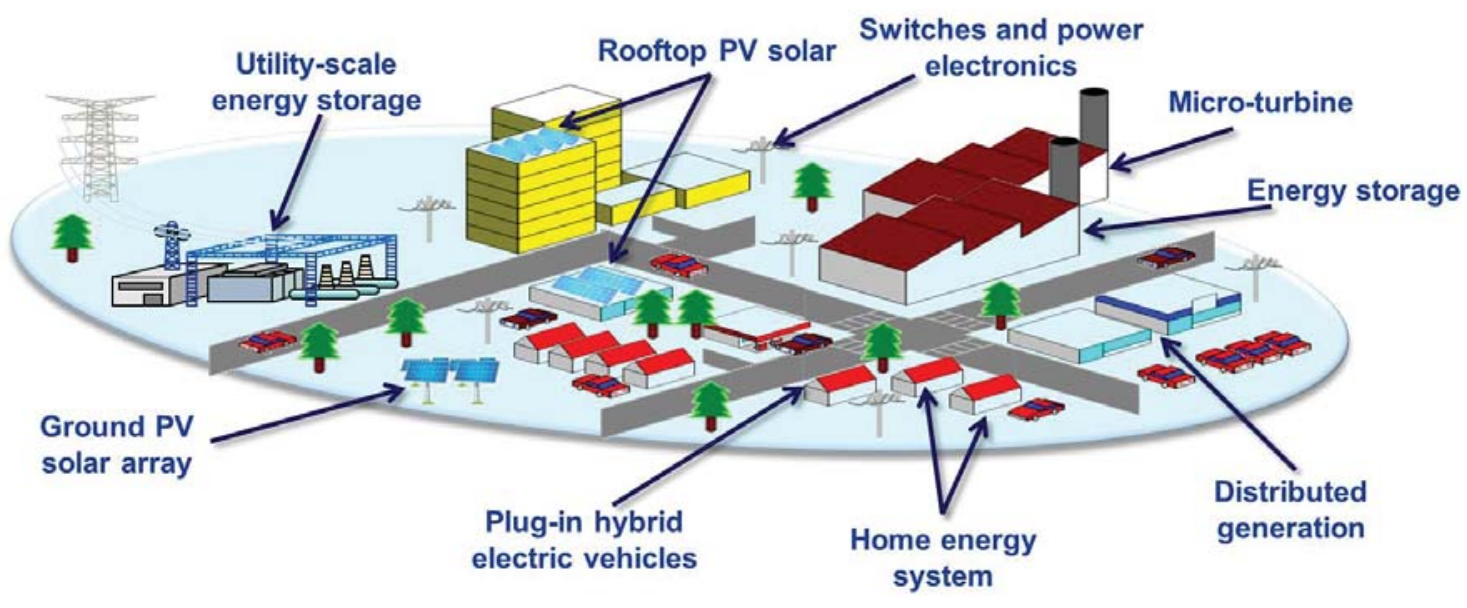

Figure 1. Schematic of microgrid

2

American Institute of Aeronautics and Astronautics 


\section{Overview of Technology Needs for Microgrid Development}

There is considerable overlap between the requirements for a terrestrial smart grid and NASA's ongoing spacecraft power system work. These overlapping requirements can be grouped into four major categories: Security, Efficiency, Accommodation of Increased Demand, and Integration. Details of these Requirements are summarized below.

1. Security

- Improve grid observability

- Rapidly detect faults and reconfigure the network to minimize brownouts / blackout

- Provide operator aids for intelligent power decision making

- Enable long term operation with minimal human intervention / operational autonomy

- Perform failure diagnostics and prognostics for power components

- Improve long term reliability / availability

2. Efficiency

- Accommodate variable/peak power demands while minimizing impact of contingencies

- Enable end-users to participate in decision making

- Manage load under variable demand and constrained capacity

- Optimize generation and distribution assets

- Automate control for operations management, fault detection, and system reconfiguration

3. Accommodation of Increased Demand

- Incorporate diverse power sources, including renewables

- Incorporate distributed energy resources

- Incorporate intelligent energy islands

- Permit incremental build-up and seamless growth

4. Integration

- Utilize common power/data interface standards

Considerable technology development is required to enable smart grid/microgrid development, for both space and terrestrial applications. The Technology Needs which must be addressed may be grouped into four development areas: Systems Technology, Simulation Technology, Automation and Controls, and Intelligent Distribution/ Hardware Integration. Some of the particular challenges in each of these areas are summarized below.

1. Systems Technology

- Routine operation of a network with a high percentage of distributed energy resources (DER)

- Demonstrate real and reactive power control using DER

- Determine the costs and benefits of distributed vs. centralized energy storage for renewables

- Understand the benefits of DC vs AC interconnections for sources and storage

2. Simulation Technology

- Develop onboard dynamic models for control algorithms

- Develop high fidelity microgrid simulation capability that can be replicated and run in real-time and faster than real-time

- Develop the ability to run hardware in the loop

3. Automation and Controls

- Develop algorithms to enable autonomous management of distributed energy resources

- Develop mechanisms to minimize generation and distribution losses

- Develop tools to enable operators to balance reliability and risk

- Determine mechanisms to compute operating margins for components, circuits, and systems

- Develop adaptive control algorithms to account for changes in plant and input parameters 
- Determine means to enable failure diagnostics

- Develop algorithms to enable automated fault recovery

- Develop prognostics to identify components that may start to exhibit faults

- Provide mechanisms to enable management of load demand

\section{Intelligent Distribution / Hardware Integration}

- Develop power electronics for bi-directional power flow techniques for real and reactive power

- Isolate faults on both sides of a grid tie inverter

- Develop secure communication network to enable the application of distributed control algorithms

- Develop and build wireless sensors

- Integrate DER with non-dispatchable generation

\section{NASA's Autonomous Control Development for Microgrid Operation}

This section covers NASA GRC's recent development work in the area of autonomous control for deep space manned vehicles, which are essentially space-based microgrids. In each section, the GRC effort is described, and the relevance to the Technology Needs described in Section III is detailed. A notional architecture for a deep space vehicle power system is described, and then an autonomous control architecture for that vehicle is presented. Next, development efforts are presented for key components of that control architecture: the Autonomous Power Controller, the Electrical Power System Simulation, and the Mission Manager/Automated Power Controller Interface. Finally a summary of the application of the NASA work to the terrestrial microgrid is discussed.

\section{A. Deep Space Vehicle Power System Architecture}

With present missions in low earth orbit, e.g. the International Space Station, the communication time between ground-based controllers and the vehicle is almost instantaneous. This allows near real-time control of spacecraft systems by ground personnel. However, as NASA missions move beyond low earth orbit, light-time communication delay issues, such as time lag and low bandwidth, will prohibit this type of operation. More distant missions will require autonomous control of manned spacecraft. To enable these missions, NASA has begun to investigate an architecture that will provide autonomous control of the spacecraft ${ }^{4,5}$ and the electrical power system in particular.

A notional power architecture for a prospective Deep Space Vehicle architecture is described in detail in Reference 6. The system features two sets of generation (solar arrays) with limited capability, four energy storage components (lithium ion batteries) which must be managed, a set of networked switchgear, and 38 electrical loads. The system also includes several docking ports, allowing the attachment of additional spacecraft, and thus the increase of the size and complexity of the electrical power system.

Note that this architecture fulfills several of the Requirements identified in Section III, including the incorporation of distributed DER (both distributed power sources and distributed energy resources), the incorporation of intelligent energy islands, and scalability.

As previously indicated, this autonomous vehicle constitutes an autonomous, islanded microgrid; a smart grid detached from any main power grid, with integrated power generation, energy storage capability, and autonomous control. The next sections will discuss NASA's ongoing work developing technology to enable this autonomous, space-based microgrid.

\section{B. Vehicle Autonomous Control Architecture}

With the power architecture specified, our next step was to define an autonomous control scheme for the deep space vehicle. The proposed communication architecture for this control scheme is shown in Figure 2, as reproduced from Reference 7. This figure provides an overall perspective on the vehicle, including high level control (Mission Manager) and multiple subsystems: controllers for the power system, the thermal system, and the Environmental Control and Life Support System (ECLSS), and associated subsystem hardware. However, as the focus of the NASA GRC effort is in power system control, this effort primarily deals with the three subsystems highlighted in green: the Mission Manager, the Autonomous Power Controller, and the electrical power system (EPS) hardware.

In this architecture, the Mission Manager (MM) works in concert with ground control and the crew to provide support mission planning, fault diagnosis, etc. Because of the long communication delays in deep space missions, the MM must also have sufficient capability to handle routine events and faults. Each of the subsystems has an autonomous controller which interfaces with the respective subsystem hardware. The controller for each subsystem 
manages local control issues and reports information and status to the MM. From the power system perspective, the most important information from the MM is a load schedule. The spacecraft's MM will work with the Autonomous Power Controller (APC) to generate a load schedule which meets mission priorities and constraints that the MM has received from the other subsystem controllers, while also satisfying the local power system constraints. As described above, the APC interfaces directly with the EPS hardware.

This control architecture addresses a number of Requirements listed in Section III. This architecture is designed to provide autonomy, enabling long term operation with minimal human intervention, while providing operator aids for intelligent power decision making in semi-autonomous mode. The architecture is also capable of managing loads under variable demand and constrained capacity, optimizing generation and distribution assets, and automating operations management, fault detection, and system configuration.

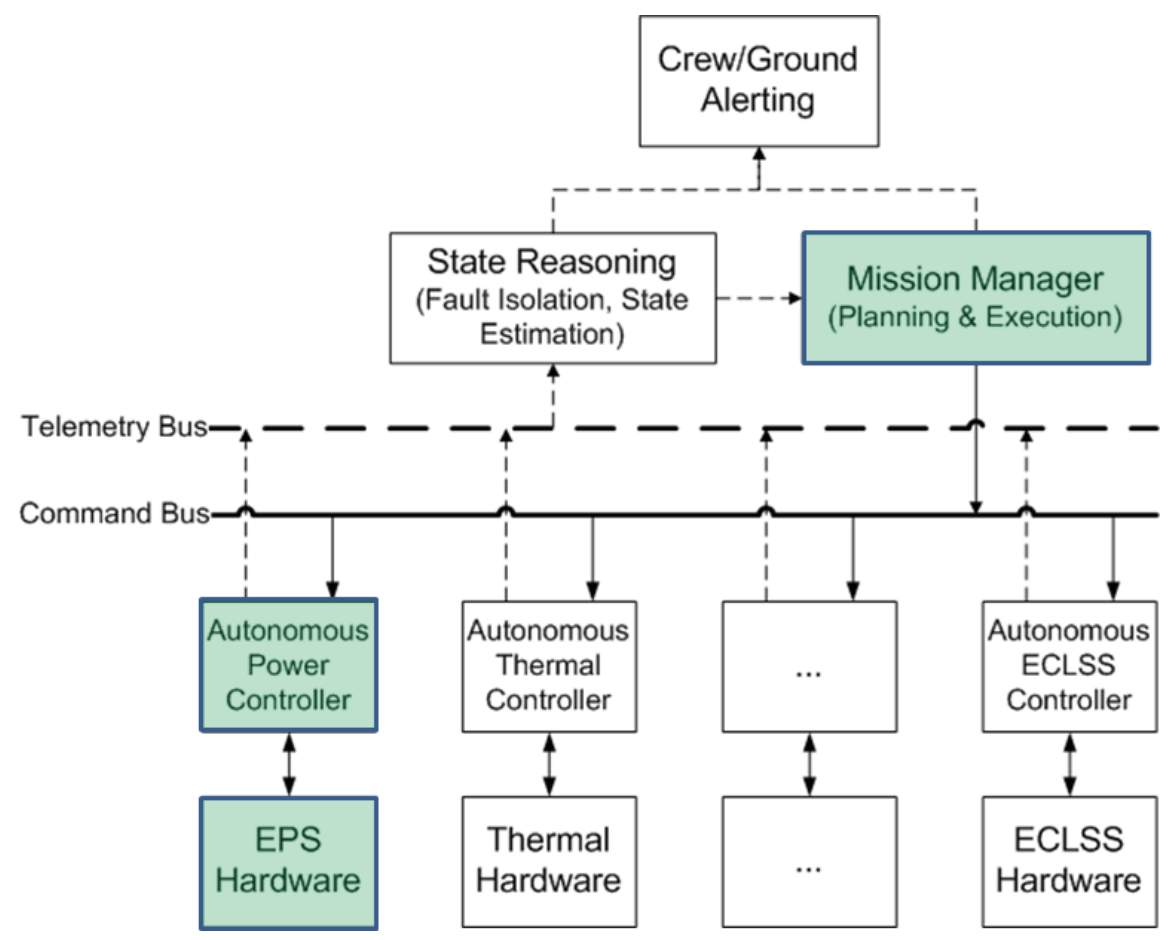

Figure 2. Communication architecture of notional autonomous spacecraft. Reproduced from Reference 7.

\section{Development of Autonomous Power Controller (APC)}

With the deep space vehicle power system and autonomous control architecture defined, our next step was to develop a control architecture that would enable autonomous operation for a vehicle electric power system. A possible architecture for the Autonomous Power Controller (APC) has been developed, described, and partially implemented, as described in Reference 8.

This APC architecture enables improved system robustness through use of a highly distributed control system. The key to the controller operation is the use of software agents that communicate in a peer-to-peer fashion to collaborate and compete with each other in order to implement the required control functions.

To enable testing, a steady-state simulation of two generic, cross-tied spacecraft electric power systems was developed in MATLAB. Each system includes generation (a solar array) and storage (a battery), and each of the loads is a constant power load with a priority as assigned by a simplified Mission Manager. During simulation, the solar arrays experienced periodic eclipses. The agents then interacted in a peer-to-peer topology to solve and implement an optimal power allocation problem under various system constraints.

This two spacecraft system was simulated for 10 hours. The testing was very successful: the control system provided maximal power to loads and ensured that the batteries are sufficiently charged, while operating with minimal data communication between agents. Thus the software agents solved a constrained optimization problem, with competing objectives, using only local information. 
This Autonomous Power Controller architecture addresses a number of Technology Needs identified in Section III, including development of algorithms to enable autonomous management of distributed energy resources, account for changes in plant and input parameters, diagnose failures and enable automated fault recovery, minimize generation and distribution losses, and enable management of load demand.

\section{Development of Electrical Power System (EPS) Simulation Library}

In order to run the above controller demonstration, a quasi-steady state, power flow-based simulation of the vehicle electrical power system (EPS) was developed and implemented. While this low fidelity model was sufficient to achieve the goals of that particular demonstration, control development requires a higher fidelity simulation environment, one which is able to capture relevant system dynamics. To this end, a library of component models for power system components is currently being developed. This library will contain hardware-validated spacecraft power system component models, and support faster-than-real-time simulation. In addition it can interface with software agents to implement autonomous controls and support the transition from the MATLAB modeling environment to hardware or hardware-in-the-loop implementation.

The library models are currently being constructed, as described in Reference 9. The library will contain detailed models of individual components, which will be combined to produce power system assemblies; and these assemblies will be combined to create end to end power system models. In order to achieve faster-than-real-time simulation, state space averaging and model reduction will be used, and Distributed Heterogeneous Simulation (DHS) integration software (PC Krause and Associates) will be used to "split" models across multiple computer processors. The model library is presently under construction, and prototype power system models have already been built. The entire model library should be completed by 4Q 2014.

The Simulation Library addresses a number of key Technology Needs identified in Section III, including the development of high fidelity simulations that can be run faster than real time, which is a key enabler for hardware in the loop operation.

\section{E. Development and Demonstration of Mission Manager / Autonomous Power Controller Interface}

For present missions, e.g. the International Space Station in low earth orbit, controllers on the ground develop a complete schedule of power usage for all spacecraft components. Several recent efforts have been focused on formalizing and automating this scheduling process using on-vehicle computation resources, as described in References 7 and 10.

The first step in demonstrating this architecture was defining the necessary communication protocol between the Mission Manager and the Autonomous Power Controller, as described in Reference 10. To this end, prototype versions of the MM and the APC were constructed, and a number of simulations were run, under a variety of operational conditions, using our nominal spacecraft architecture. This enabled development of content and format of the messages necessary to achieve the desired goals: negotiation of a load schedule that meets the global requirements (contained in the MM) as well as the local power system requirements (contained in the APC), and communication of off-plan disturbances that arise while executing a negotiated plan. The message content was developed in two steps: first, a set of rapid-prototyping "paper" simulations were preformed, to ensure that concise yet thorough message content was defined; then the resultant optimized messages were codified for computer communication for use in automated testing.

Next, in order to determine the feasibility of this architecture and to validate its capability to respond to both disturbances and changing constraints in an appropriate manner, a computer-based simulation was conducted, using the system configuration described in Reference 7. Automated versions of the MM and the APC were developed and run in two different physical locations: the automated MM at NASA Ames Research Center in Mountain View, CA, and the APC at NASA Glenn Research Center in Cleveland, OH. The two systems communicated via TCP Sockets over the Space Network Research Federation (SNRF) dedicated network. In order to test the communication, the quasi-steady state, power flow-based simulation of the notional vehicle electrical power system (EPS) is used, also running on at NASA GRC, and, also via the SNRF network, loads are powered on or off at NASA Johnson Space Center in Houston, TX.

This effort addresses a number of Technology Needs identified in Section III, including the development of data interface standards, the development of communication networks to enable the application of distributed control algorithms, and the enabling of high fidelity model use and hardware in the loop operation.

\section{F. Summary of GRC Microgrid Application to Terrestrial Microgrids}

The microgrid work done at NASA is readily applicable to terrestrial microgrids. Firstly, the control architecture described above is similar to the operational procedure that is used in terrestrial power grid operation centers ${ }^{6}$. Also 
space-based microgrids contain all of the elements necessary for a terrestrial microgrid, e.g. DER, a distribution network, a collection of prioritized loads, and an autonomous power control system. While the spacecraft autonomous power controller is controlling a comparatively small DC electrical power system, the lessons learned and control techniques developed are almost directly applicable to the AC terrestrial system, contributing to a number of the defined Technology Needs areas. Further, by first implementing this control on a spacecraft there is a minimum of historical legacy to overcome, and the experience gained can inform terrestrial development by serving as a test bed.

\section{GRC Physical Assets for Microgrid Technology Development}

The West Area of NASA GRC is referred to as the GRC Power Campus. The majority of the space-based power system research and development work is performed in this area. The power campus has approximately 2MW of loads, and is comprised of six major facilities that support a wide range of power related research, development, and testing: the Space Power Research Laboratory, the Fuel Cell Testing Facility, the Electric Power Laboratory, the Energy Conversion Laboratory, the Structural Assembly Building, and the Power Systems Facility. An overview of the facilities, along with a summary of the work they support, is shown in Figure 3. Details of the capabilities of each of these facilities are presented below.

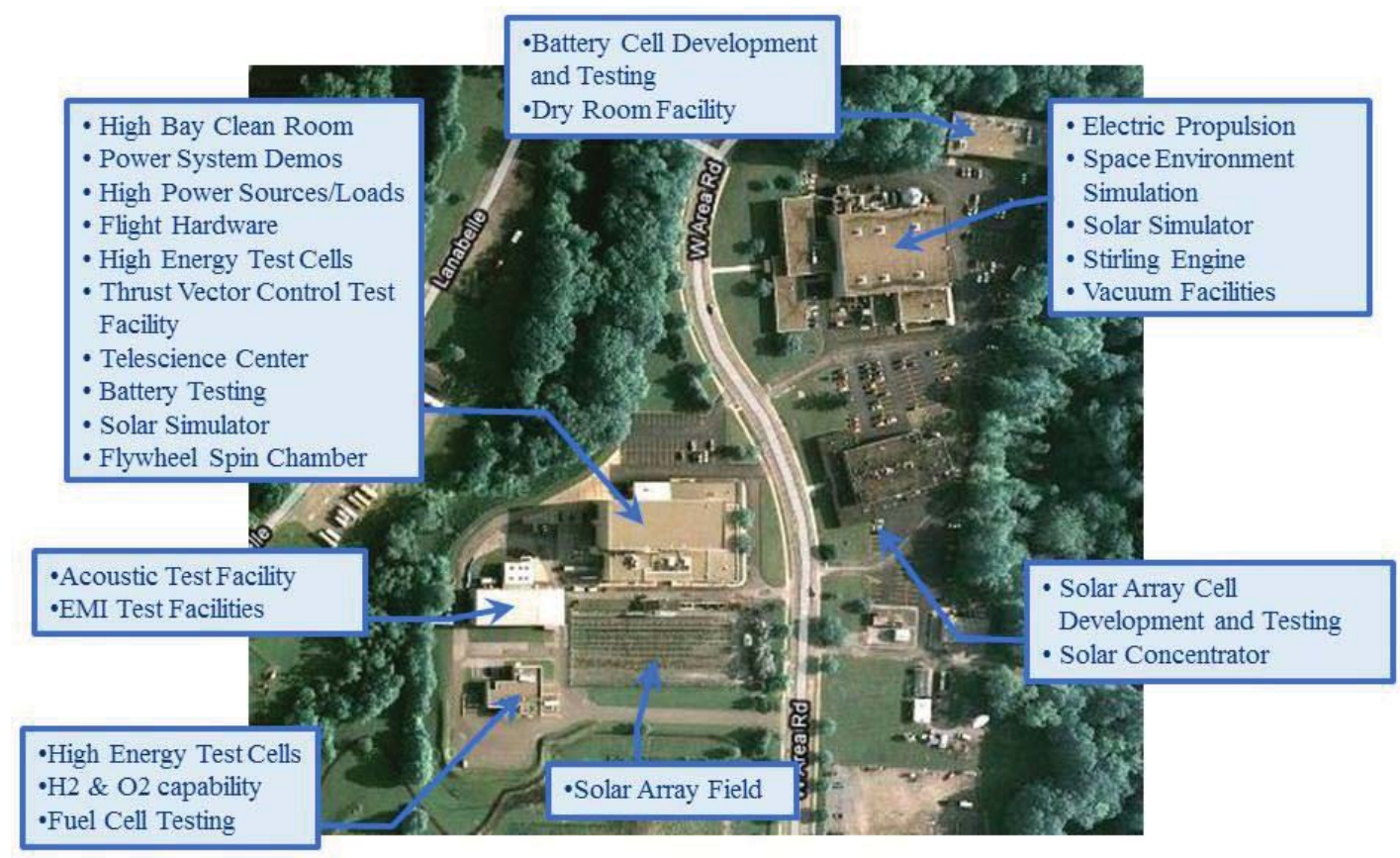

Figure 3. Aerial view of GRC Power Campus

The Space Power Research Laboratory supports battery cell development and testing, along with fuel cell development. Battery research areas include lithium-ion, lithium-air, lithium-oxygen, and lithium polymer; alternate chemistries include nickel-hydrogen, sodium-sulfur, and other advanced chemistries.

The Fuel Cell Testing Facility provides three test cells that can support pure hydrogen and oxygen fuel cell systems, and other high energy power generation technologies. Fuel cell development areas include proton exchange membrane systems, solid oxide systems, and regenerative systems.

The Electric Power Laboratory has several unique vacuum facilities. These vacuum facilities support a variety of space electric propulsion testing, along with Stirling engine development and testing.

The Energy Conversion Laboratory supports the research and development of advanced photovoltaic systems including solar cells, radioisotope thermophotovoltaics, and concentrated solar and advanced solar power deployment systems. Additionally, two power generating wind turbines are also located adjacent to the Energy Conversion Laboratory. 
The Structural Assembly Building houses both an acoustic test facility, and Electromagnetic Interference (EMI) Laboratory test facilities to qualify power systems for space flight. The EMI lab offers several Electromagnetic Compatibility (EMC) services like EMC hardware design to meet the desired requirement, specification comparisons, consultation during design development, and prefabrication analyses. This facility consists of three shielded rooms, two test chambers, and a control room.

The Power Systems Facility (PSF) has a large Class 100,000 high bay cleanroom, and is designed to support large power system design and development projects; the International Space Station power system was designed and developed in this facility. PSF contains test cells capable of supporting high energy battery and advanced energy storage flywheel testing. Solar arrays and dynamic solar array simulators, along with a variety of high power DC power sources and loads, are available for testing. An autonomous power system laboratory and the Telescience Support Center are also located in PSF.

The close proximity of all of these power system development resources provides a unique capability to support the development of advanced microgrid systems. Future large space based power systems will share many of the attributes and challenges that terrestrial microgrids have. Much of the infrastructure is in place at the NASA GRC Power Campus to allow the integration of all of the power generation and storage systems into a microgrid. This capability to support integrated system level testing will be invaluable in the development of complex multi-source microgrids, as well as the necessary autonomous controls necessary to make microgrids stable, robust elements of the United States power grid.

The GRC Power Campus presently receives utility power from an electrical substation that is dedicated to the GRC Power Campus buildings. This would allow the microgrid test capability to expand to include the Power Campus facilities as part of the development capability. With the addition of base power generation capabilities the entire Power Campus would be able to support the development and testing of a microgrid having a substantial percentage (25\%-50\%) of renewable power sources as part of the utility.

\section{Phased Development of the GRC Microgrid Test Facility}

NASA GRC has considerable capabilities which can be brought to bear in developing a microgrid test facility. NASA's experience in the power field, including the development of autonomous spacecraft power systems, planetary surface power, and the International Space Station (ISS) power system provides a unique perspective on this problem, and an opportunity for "clean sheet" approaches to the implementation of terrestrial microgrids. The NASA team's extensive experience (40+ years) in the development of end-to-end power systems with renewable sources and storage (including ISS, Space Solar Power, Launch Vehicles, Advanced Aero Vehicles, etc.), combined with a strong analytical component (controls and simulation anchored with integrated hardware), provides a sound basis for understanding and managing the issues associated with the operation of a microgrid dominated by renewables. Additionally, the geography of the NASA Power Campus provides the opportunity for the isolation of the microgrid from the main power grid, resulting in a unique testing facility which enables "edge of the envelope" testing and rapid evaluation of innovative control and management strategies.

The objectives of the GRC Microgrid Test Facility are to demonstrate the long term, automated, stable operation of a microgrid at the GRC Power Campus that includes renewable energy sources, energy storage systems, advanced power distribution control and automation, wireless data communication capability, and net metering of energy sources and loads. The microgrid could be decoupled from the main grid, providing a subscale system test platform for new technology development and deployment, with the added advantage of providing results applicable to both terrestrial and space systems. The facility would enable easy integration of new technology demonstrators into the grid for system level demonstrations, and provide opportunities to understand the integration and interface issues with the development of power grids with a high percentage of renewable generation and distributed energy storage, and to evaluate AC vs DC microgrid interconnection strategies.

The proposed development approach would leverage capabilities that already exist on the Power Campus at NASA GRC. First, high fidelity simulation models would be developed and verified; then these models would be demonstrated running as hardware in the loop, to extend the results to larger systems. Next, renewable generation, distributed energy storage, and intelligent loads would be integrated on an AC and / or DC microgrid, and bidirectional power flow techniques for both real and reactive power would be demonstrated. Finally, advanced distributed power control algorithms that guarantee stability (angle, frequency, and voltage) and operational security would be developed and demonstrated.

The phased development of the GRC Microgrid Test Facility is defined below. 
Phase I

1) Demonstrate limited operation and control of the interconnected solar arrays, wind turbines, fuel cells, and storage devices

2) Demonstrate distributed SCADA control of the microgrid

3) Manually control power flow, voltage, and power quality from different sources and storage devices

4) Develop high fidelity simulation models of the microgrid

5) Acquire data and validate simulation models

6) Develop normal mode operation autonomous controls and verify them with simulation models

7) Evaluate alternative energy provider hardware with limited microgrid control

Phase II

1) Demonstrate hardware in the loop operation with simulation models and microgrid hardware

2) Demonstrate normal mode autonomous controls with simulation models / microgrid hardware

3) Demonstrate normal mode operation that guarantees stability (angle, frequency and voltage) and operational security

4) Demonstrate normal mode operation for bi-directional power flow techniques for real and reactive power using storage

5) Develop failure mode operation autonomous controls and verify them via simulations

6) Demonstrate failure mode autonomous controls with simulation models / microgrid hardware

7) Evaluate alternative energy provider hardware with complete autonomous microgrid control and hardware

\section{Conclusion}

This paper describes NASA GRC's recent work in the development of autonomous deep space manned vehicles, which are islanded, space-based microgrids, and explains how this work can be applied to the development of terrestrial microgrids. A discussion of the benefits of microgrid use within the changing power grid is provided, as is an overview of the Requirements and Technology Needs which must be met to enable space-based and terrestrial microgrids. GRC's work in the area of autonomous controller development for space vehicles is described, including development of a deep space vehicle power system architecture, autonomous control architecture, autonomous power controller, electrical power system simulation, and key automated controller subsystems (including the Mission Manager, the Autonomous Power Controller, and their communication interface protocol); and the contribution of each of these developments on the microgrid Technology Needs is detailed. Finally the unique, diverse set of GRC physical assets which can be applied to microgrid development was presented, along with a plan for phased development of a GRC Microgrid Test Facility.

Ongoing work in this area includes the completion of the Electrical Power System (EPS) Simulation Library and the phased development of the GRC Microgrid Test Facility.

\section{Acknowledgments}

The authors would like to thank the NASA Advanced Exploration Systems Modular Power Systems Project for funding this work.

\section{References}

${ }^{1}$ Amin, S.M., and Wollenberg, B.F., "Toward a Smart Grid: Power Delivery for the 21 st Century", IEEE Power and Energy Magazine, September-October, 2005.

${ }^{2}$ Ipakchi, A., and Albuyeh, F., "Grid of the Future", IEEE Power and Energy Magazine, March-April 2009.

${ }^{3}$ Dohn, R.L., "The Business Case for Microgrids", Siemens.com Global Website [White Paper], URL: http://www.energy. siemens.com/us/pool/us/energy/energy-topics/smartgrid/downloads/The\%20business\%20case\%20for\%20microgrids_Siemens \%20white\%20paper.pdf [cited 26 June 2014].

${ }^{4}$ J. Frank, L. Spirkovska, R. McCann, L. Wang, K. Pohlkamp, L. Morin, “Autonomous Mission Operations,” 2013 IEEE Aerospace Conference, Big Sky, MT, March 2-9, 2013.

${ }^{5}$ S. Colombano, L. Sprikovska, V. Baskaran, G. Aaseng, R. McCann, J. Ossenfort, I. Smith, D. Iverson, and M. Schwabacher, "A system for fault management and fault consequences analysis for NASA's Deep Space Habitat," AIAA SPACE 2013 Conference and Exposition, September 2013.

${ }^{6}$ Soeder, J.F., Beach, R., McNelis, N., McNelis, A., Dever, T., May, R.D., "Overview of Intelligent Power Systems Development for Human Deep Space Exploration", to be published at IECEC, Cleveland, OH, July 28-30, 2014. 
${ }^{7}$ May, R.D., Dever, T.P., Soeder, J.F., George, P.J., Morris, P.H., Colombano, S.P., Frank, J.D., Schwabacher, M.A., Wang, L., Lawler, D., "An Architecture to Enable Autonomous Control of Spacecraft", to be published at IECEC, Cleveland, OH, July 28-30, 2014.

${ }^{8}$ May, R.D., Loparo, K.A., "The Use of Software Agents for Autonomous Control of a DC Space Power System," to be published at IEEE EnergyTech 2014, Cleveland, OH, July 28-30, 2014.

${ }^{9}$ McNelis, A., Beach, R., Soeder, J., McNelis, N., May, R., Dever, T., Trase, L., "Simulation and Control Lab Development for Power and Energy Management for NASA Manned Deep Space Missions," to be published at IECEC, Cleveland, OH, July 28-30, 2014.

${ }^{10}$ Dever, T.P., May, R.D., Morris, P.H., “Autonomous Spacecraft Communication Interface for Load Planning”, NASA/TM2014-218362, 2014. 